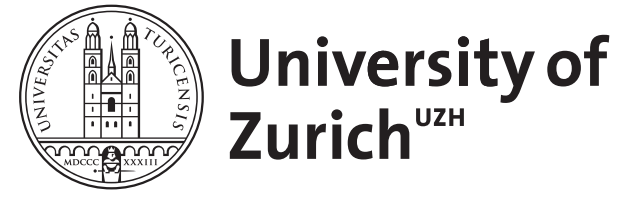

Beziehungsanbahnung im Netz. Text, Bild und Gatekeeping

Dürscheid, Christa

DOI: https://doi.org/10.1515/9783110496918-004

Posted at the Zurich Open Repository and Archive, University of Zurich

ZORA URL: https://doi.org/10.5167/uzh-132257

Book Section

Published Version

Originally published at:

Dürscheid, Christa (2017). Beziehungsanbahnung im Netz. Text, Bild und Gatekeeping. In: Linke, Angelika; Schröter, Juliane. Sprache und Beziehung. Berlin: De Gruyter, 49-71.

DOI: https://doi.org/10.1515/9783110496918-004 


\title{
Christa Dürscheid \\ Beziehungsanbahnung im Netz
}

\author{
Text, Bild und Gatekeeping
}

\section{Zur Einführung}

Das Internet bietet schier unendliche Möglichkeiten - bekanntlich auch für die Partnersuche. Immer wieder liest man Berichte über Paare, die sich über das Internet kennengelernt haben, und längst ruft es keine Irritation mehr hervor, wenn man im Freundeskreis davon spricht, dass man im Internet nach einem Partner sucht bzw. - vorsichtiger gesagt - dass man das Internet dazu benutzt, um neue Kontakte zu knüpfen, die in eine Paarbeziehung münden könnten. Ob dieser Weg tatsächlich so erfolgreich ist, wie einige Zeitungsartikel über das Thema nahe legen, sei dahingestellt; die Medienberichte zeigen ein divergentes Bild. So heißt es einerseits in einem Artikel der Neuen Zürcher Zeitung vom 28. Juli 2010, dass „über 14 Prozent der Beziehungen [...] einer digitalen Kontaktaufnahme“ entstammen, andererseits ist auf Spiegel Online vom 10. November 2013 unter dem Titel „Die virtuelle Liebesfalle“ zu lesen, dass „die Kraft des OnlineDatings maßlos überschätzt“ werde und sich unter den befragten Paaren, so zeige das Ergebnis einer repräsentativen Allensbach-Studie, nur gerade „mal zwei Prozent über Single-Portale im Netz “ kennengelernt hätten. ${ }^{1}$

Liest man solche Artikel, dann stellt sich die grundsätzliche Frage, auf welcher Basis die genannten Zahlen erhoben wurden und wer die jeweiligen Auftraggeber der in den Artikeln genannten Studien sind. Das aber steht hier nicht zur Debatte; im vorliegenden Beitrag interessieren aus linguistischer Sicht andere Fragen. Es sind dies die folgenden: Welche Faktoren spielen eine Rolle, wenn die Courtship-Kommunikation ${ }^{2}$ ihren Anfang nicht in der Offline-, sondern in der Online-Welt nimmt? Wie gestaltet sich die Beziehungsanbahnung über Online-

1 Die Artikel sind online verfügbar unter: http://www.nzz.ch/aktuell/panorama/partnersucheim-internet-gewinnt-an-bedeutung-1.6951240 und http://www.parship.de/pics/downloads/de DE/Bericht_PARSHIP-Paarbefragung_2012.pdf [22.6.2016].

2 An dieser Stelle sei nur eine kurze Definition gegeben (vgl. dazu ausführlich Abschn. 3): Unter Courtship wird der „Teil der interpersonalen Kommunikation verstanden, der dem Aufbau intimer Beziehungen zwischen zwei Personen dient“ (Guhr 2008: 18). 
Partnervermittlungsagenturen in sprachlicher Hinsicht? Wo liegen die Unterschiede zur Kommunikation in sozialen Netzwerken wie z. B. Facebook, wo es ja auch vorkommen kann, dass man über diese Plattform Kontakte mit anderen knüpft, die man nicht persönlich kennt? Das ist z. B. der Fall, wenn man auf Facebook eine „Freundschaftsanfrage“ verschickt, man aber die Person, welche diese Anfrage erhält, nur über Dritte kennt. Zwar sind die Nutzungsmotive auf Facebook in der Regel andere als bei einer Online-Partnervermittlung (vgl. dazu Abschn. 2), doch gilt für beide Plattformen, dass die Kontaktaufnahme schriftlich erfolgt, dass auch der weitere Austausch zunächst schriftbasiert ist und dass die Sprache in beiden Kontexten eine wichtige beziehungsstiftende Funktion hat. Denn nur über die Sprache gelingt es in der Online-Welt, mit einer fremden Person in Kontakt zu treten, die man kennenlernen möchte. Ein Blickkontakt, ein Lächeln, eine freundliche Geste - all das ist weder auf Facebook noch auf den Plattformen von Partnervermittlungsagenturen möglich.

Im Folgenden werde ich die Möglichkeiten der Erstkontakt-Aufnahme auf einer dieser Websites, auf Parship (vgl. www.parship.de), näher betrachten. Dabei handelt es sich um eine Partnervermittlung, die im Jahr 2000 gegründet wurde und heute neben ElitePartner (vgl. www.elitepartner.ch) zu den umsatzstärksten Online-Anbietern im deutschsprachigen Raum zählt. Natürlich gibt es noch weitere Partnervermittlungsagenturen, die im Netz ihre Dienste anbieten (vgl. www.singleboersen-vergleich.ch), hier aber nicht im Fokus stehen. In

Zuletzt online: 24.07 .14
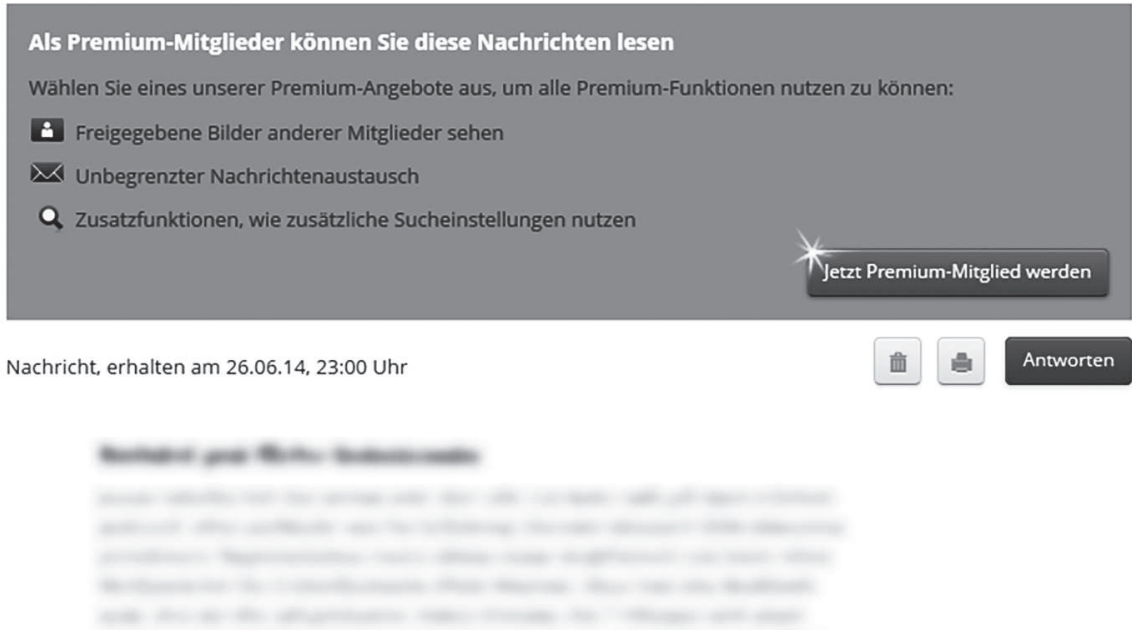

Abb. 1: Von Parship verschickte Mitteilung. 
der Regel besteht die Möglichkeit, diese Angebote gratis zu testen, d. h. bei dem jeweiligen Anbieter ein eigenes Profil einzurichten, ohne einen monatlichen Mitgliedsbeitrag zu zahlen. Doch spätestens dann, wenn man die Nachricht eines potentiellen Interessenten erhält, muss man Mitglied werden, um die Mitteilung lesen zu können. Das veranschaulicht die obenstehende Abbildung. Strategisch geschickt wird der Adressat ${ }^{3}$ hier nicht nur darauf hingewiesen, dass eine Nachricht für ihn vorliegt, der Text wird auch angezeigt - allerdings so, dass der Empfänger ihn nicht lesen kann, da er kein zahlendes Mitglied ist.

Die meisten Online-Kontaktbörsen wenden sich an bestimmte Zielgruppen. Eine große Zielgruppe sind die Senioren (vgl. z. B. http://zweiterfruehling.ch). Daneben gibt es aber auch kleinere Internetportale, die ihr Angebot auf Personen mit einer bestimmten religiösen oder politischen Einstellung ausrichten. ${ }^{4}$ Dagegen will Parship keine spezifische Zielgruppe ansprechen; die Agentur wendet sich, so heißt es auf ihrer Website, generell an „seriöse Singles“ - im Gegensatz z. B. zu ElitePartner, wo bereits im Logo damit geworben wird, dass dies eine Partnervermittlung für „Akademiker \& Singles mit Niveau“ sei (vgl. www.elitepartner.de). Beiden Plattformen gemeinsam ist, dass sie sich an Personen richten, die eine feste Partnerschaft suchen (anders als dies z. B. in Single-Chats oder Kontaktanzeigenportalen der Fall ist), und dass sie schriftbasiert sind. Was den letztgenannten Punkt betrifft, so unterscheiden sie sich darin von Kontaktbörsen, über die man sich im Internet zwar anmelden kann, die Kontaktaufnahme selbst aber mündlich, in einer Face-to-Face-Situation erfolgt (z. B. beim Speed-Dating oder in Freizeitclubs für Singles). Doch darauf sei hier nur am Rande verwiesen; im Folgenden liegt der Schwerpunkt auf solchen Varianten der Partnersuche, in denen die Beziehungsanbahnung zum einen schriftlich, zum anderen via Internet (also nicht z. B. über eine Zeitungsannonce) erfolgt. Dass in einem solchen Kontext der Text und das Bild eine wichtige Funktion einnehmen, ist naheliegend. Der Titel des vorliegenden Beitrags weist denn auch auf die Relevanz dieser beiden Faktoren in der Online-Partnersuche hin. Allerdings mag es erstaunen, dass im Titel von „Gatekeeping“ die Rede ist. Deshalb sei hier eine kurze Erklärung gegeben. Zunächst zum Terminus:

Als „Gatekeeper“ (dt. Torwächter) wird eine Person bezeichnet, die darüber entscheiden kann, ob jemand einen Durchgang passieren darf oder nicht. In der

3 Eine Geschlechterdifferenzierung wird in diesem Beitrag nicht vorgenommen. Aus der maskulinen Form darf daher nicht auf das Geschlecht geschlossen werden.

4 So führt eine Suchabfrage mit den Stichwörtern „Partnersuche“ und „christlich“ auf die Website http://www.partnersuchechristlich.ch, auf der Mitglieder registriert sind, die „deinen Glauben teilen und ihren Seelenverwandten finden wollen“ [22.9. 2016]. 
Publizistik wird der Terminus auf Nachrichtenjournalisten bezogen: Sie entscheiden (z. B. aufgrund ihrer persönlichen Vorlieben oder mit Blick auf die jeweilige Zielgruppe), welche Informationen den Weg in die Öffentlichkeit finden - und welche nicht. ${ }^{5}$ Doch längst sind es nicht mehr nur Nachrichtenjournalisten, die eine solche Gatekeeping-Funktion inne haben. Auch Suchmaschinen selektieren die Flut an Informationen, ebenso können z. B. Twitter-User, die über ein politisches Ereignis berichten, diese Funktion wahrnehmen, indem sie aus der Menge an bereits vorhandenen Medienberichten zu einem Thema diejenigen auswählen, die sie kommentieren oder auf die sie mit einem Link verweisen (vgl. zu diesem ,audience gatekeeping“ Kwon et al. 2012). Das Gatekeeping lässt sich also nicht nur auf die „Selektionsfunktion von Nachrichtenjournalisten“ (Beck 2013: 100) beziehen, es findet auch in anderen Bereichen Anwendung - so auch in der Online-Partnersuche. Anders als im Journalismus ist es hier aber nicht der Schreiber, der aus der Flut an Informationen eine Auswahl trifft, sondern der Leser, der aufgrund der Darstellung auf der Profilseite entscheidet, ob er mit der Person Kontakt aufnehmen möchte. Ein wichtiges Kriterium in diesem Selektionsprozess ist, wie sich der Text präsentiert und wie sehr das Profilbild, d.h. das Foto der betreffenden Person, gefällt. Mit anderen Worten: In der Online-Partnersuche haben Text und Bild (sofern Letzteres nicht gepixelt oder mit einem Platzhalter versehen ist) eine wichtige Funktion. Sie dienen der Person, die das Gatekeeping vornimmt, als Entscheidungsgrundlage.

Diesen Punkt werde ich weiter unten, in Abschn. 4, genauer ausführen, zunächst geht es nun um die Unterschiede und Gemeinsamkeiten zwischen Online-Partnervermittlungen und sozialen Netzwerken (vgl. Abschn. 2). Denn auch Letztere können der Beziehungsanbahnung dienen, also dazu verwendet werden, „romantische Erstkontakte“ (Marx 2012a: 49) zu knüpfen (vgl. dazu auch Frick 2014). Im Anschluss daran werde ich verschiedene Kommunikationspraktiken auf Parship genauer beschreiben und im Kontext der Courtship-Kommunikation verorten (vgl. Abschn. 3). Allerdings liegen zur Courtship-Kommunikation bisher nur wenige Forschungsarbeiten vor, die zudem alle davon ausgehen, dass eine Person die werbende ist (d.h. ,den Hof macht') und die andere die umworbene. Dagegen basiert Parship auf dem Prinzip der beidseitigen Partnerwerbung. Die Akteure haben einen gemeinsamen Erwartungshorizont; sie sind gleichzeitig Werbende (auf ihren Profilseiten) und Umworbene (als Leser der Profilseiten

5 Vgl. hierzu die Erläuterung im „Lexikon Kommunikations- und Medienwissenschaft“ (unter dem Eintrag Gatekeeper): „Der einzelne Journalist ist Träger einer spezifischen Berufsrolle, zu der die Selektion und Verarbeitung von Nachrichten nach bestimmten, empirisch belegbaren Regeln gehört“ (Beck 2013: 100). 
anderer). Es handelt sich also um einen spezifischen Typus von Courtship-Kommunikation, die zudem schriftlich ist und nicht, wie im prototypischen Fall, faceto-face erfolgt. Welche Rolle die schriftlichen Ausdrucksfähigkeiten in diesem Setting spielen (und hier insbesondere die Orthographie), wird in Abschn. 4 diskutiert. Abschn. 5 schließlich zeigt auf, dass es in der linguistischen Forschung zur Online-Partnersuche noch viele offene Fragen gibt, die auf empirischer Grundlage untersucht werden müssen.

\section{Parship und Facebook im Vergleich}

Wie bereits erwähnt, gibt es Parallelen zwischen Parship und Facebook. In beiden Fällen handelt es sich um eine schriftbasierte Kommunikation; in beiden Fällen kann die Kommunikation zur Beziehungsanbahnung dienen, auch wenn dieses Motiv in der Regel nur bei Parship offengelegt wird. Facebook ist zwar nicht auf die Partnervermittlung ausgerichtet; da ein User aber auf seiner Profilseite den Beziehungsstatus angeben kann (,Single“, „Verlobt“, „Verheiratet“, „in einer offenen Beziehung“, „Es ist kompliziert“, „Getrennt“, „Geschieden“, „Verwitwet“), findet jemand, der auf Partnersuche ist, hier eine wichtige Information (vgl. dazu auch Schibli 2014: 4). Wird als Beziehungsstatus z. B. „Single“ oder „Geschieden“ angegeben, kann dies dem Profilbesucher signalisieren, dass potentiell eine „Ansprechbarkeit“ gegeben ist (vgl. dazu Marx 2012a: 57). Doch ist es auch möglich, wie Karina Frick (2014) in ihrer Arbeit zum Thema „Liebeskommunikation über Facebook“ betont, auf eine Angabe zu verzichten, d. h. keine der vorgegebenen Optionen anzuklicken. So weist Konstanze Marx denn auch darauf hin, dass in ihrer Stichprobe von 100 Facebook-Nutzern nur sieben Profile mit einer Angabe zum Beziehungsstatus versehen waren und sich darunter keine fanden, auf denen beispielsweise „Single“ vermerkt war (vgl. Marx 2012a: 56).

Was die Möglichkeiten der Kontaktaufnahme auf Facebook und Parship betrifft, so sind diese ebenfalls vergleichbar: Auf beiden Plattformen kann man persönliche Nachrichten verschicken oder der anderen Person ein „Lächeln“ (auf Parship) bzw. einen „Anstupser“ (auf Facebook) zukommen lassen. Bei beidem handelt es sich um einen Befehl, der nur durch einen Klick eingegeben wird; eine Texteingabe ist nicht erforderlich. Der andere erhält daraufhin eine Benachrichtigung, dass ihm ein Lächeln geschickt bzw. dass er angestupst wurde. Solche Nachrichten stehen als kleine, non-verbale Beziehungsbotschaften in einem Handlungsrahmen, der, wie Androutsopoulos (2007: 75) schreibt, „ohne die aus der direkten verbalen Interaktion vertrauten Mittel“ auskommen muss. Das „Lächeln“ oder der „Anstupser“ sollen hier also das zum Ausdruck bringen, was 
in der Offline-Welt (oder in einer Videokommunikation) beispielsweise über die Mimik oder die Gestik angezeigt werden kann. ${ }^{6}$ Zudem kann man auf Parship ein so genanntes „Kompliment“ verschicken, also beispielsweise dem anderen über eine vom System generierte Nachricht mitteilen, dass man sein Profilbild gut findet. Diese Möglichkeit ist vergleichbar mit dem „Gefällt mir“-Button, der auf Facebook, aber auch auf anderen Plattformen zu finden ist - mit dem Unterschied, dass sich die über Parship verschickten Positivbewertungen immer auf eine Person (z. B. ihr Foto) und nicht auf eine Sache beziehen. Das überrascht nicht: Alle von Parship vorgeschlagenen Aktivitäten dienen der Beziehungsanbahnung; das ist der kommunikative Zweck dieser Plattform. So kann man einen Fragebogen ausfüllen, der dem System dazu dienen soll, geeignete Partnervorschläge zu machen. Man kann sich in einem Diskussionsforum von anderen Mitgliedern Rat zu verschiedenen Fragen rund um die Partnersuche holen. Oder man kann jemandem eine sog. „Spassfrage“ stellen, d. h. einen von Parship vorgegebenen Satz auswählen, den der andere zu Ende führen soll (z. B. „Wenn ich jemanden kennen lerne, der eine Heirat für sich ausschließt ...“). In Abb. 2 sind einige dieser Optionen zusammengestellt.

Wie die Textauszüge in Abb. 2 zeigen, wird hier der Adressat gesiezt (z. B. „Ein Lächeln für Sie“). Auf Facebook ist das anders; der User wird vom System konsequent mit $\mathrm{Du}$ adressiert (z. B. „Besuche den Hilfebereich oder melde ein Problem“). In beiden Fällen handelt es sich um vom System verfasste Mitteilungen; wie sieht es demgegenüber mit der Wahl der Anredeform aus, wenn die Akteure selbst die Nachrichten schreiben? Die Vermutung liegt nahe, dass auch dann die Anrede in der 2. Person Singular oder 2. Person Plural bevorzugt wird. Das zeigt sowohl ein rascher Blick auf die Pinnwand von Facebook-Usern (z. B. „Hey, Leute, kennt ihr das?“) als auch auf die Profilseiten auf Parship (z. B. „Du spürst, was ich denk!!“). Im zweiten Fall handelt es sich um das Originalzitat eines Parship-Mitglieds. ${ }^{7}$ Das $D u$ richtet sich hier nicht an eine bestimmte Person, sondern an einen imaginären Partner. Diese Variante der Du-Anrede ist nicht zu verwechseln mit dem generischen $D u$, das v. a. in der gesprochenen Sprache vorkommt (vgl. dazu Stukenbrock in diesem Band) (vgl. Wenn du im Beruf Erfolg haben willst, dann musst $d u$...). Dieses Du kann im Deutschen unter bestimmten Bedingungen an die Stelle des Indefinitpronomens man treten (vgl. Wenn man

6 Nicola Döring (2009: o.S.) schreibt dazu: „[D]amit wird ähnlich wie beim Blickkontakt wortlos wechselseitiges Interesse erkennbar. Manche Plattformen bieten auch die Möglichkeit, virtuelle Rosen oder Küsse zu verschenken.“

7 Alle Beispiele, die ich hier anführe, stammen von Personen, die auf Parship angemeldet waren und mir ihre Daten zur Verfügung gestellt haben. 


\section{PARSHIP}

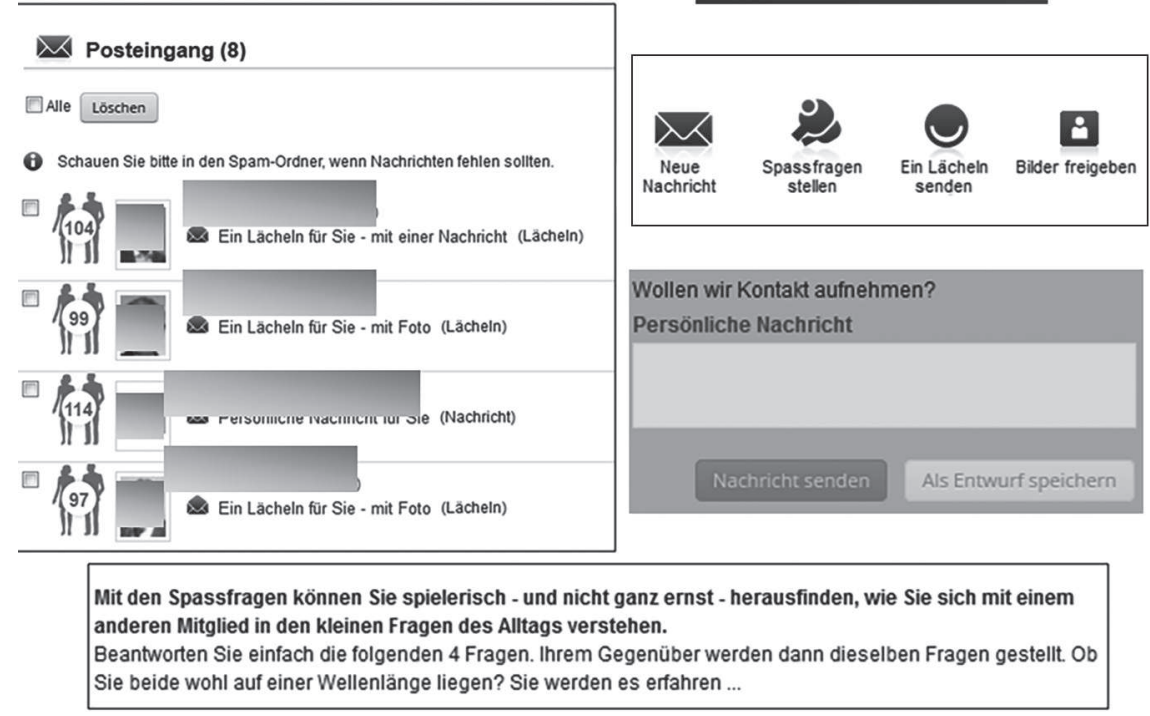

Abb. 2: Kommunikative Aktivitäten auf Parship.

im Beruf Erfolg haben will, dann muss man ...). Das gilt für das an eine imaginäre Person gerichtete $\mathrm{Du}$ nicht, eine Formulierung wie Man spürt, was ich denk! ist in diesem Kontext nicht möglich.

Neben diesem $\mathrm{Du}$, das sich an einen Wunschpartner richtet, von dem man aber noch gar nicht weiß, ob es ihn gibt, gibt es noch eine dritte Variante des Du-Gebrauchs: Wie Abb. 2 zeigt, kann auf Parship anstelle eines „Lächelns“ oder einer „Spassfrage“ eine persönliche Nachricht geschickt werden. Und auch hier kommt es vor, dass die Du-Anrede gewählt wird, obwohl man den Empfänger nicht kennt. Auf diese Weise soll über die Anrede eine Nähe zum Adressaten hergestellt werden, die es zu diesem Zeitpunkt noch gar nicht geben kann. Die Nähesprachlichkeit resultiert hier also nicht aus der Vertrautheit der Kommunikationspartner, sie soll diese Vertrautheit inszenieren bzw. bereits antizipieren (da es ja sein könnte, dass man sich näher kommen wird). Mit anderen Worten, in Anlehnung an Androutsopoulos (2007: 80): Allein durch die ,strategische Sprachgestaltung “ kann eine Situation der Distanz symbolisch als eine Situation der Nähe aufgeladen werden. Dies sei im Folgenden an zwei Nachrichten illustriert (in Originalschreibung), die über Parship verschickt wurden und aus einer kleinen privaten Textsammlung stammen, welche mir zur Verfügung gestellt 
wurde. ${ }^{8}$ Wie man in (2) sieht, wird der Adressat hier nicht nur geduzt, der Schreiber verwendet auch eine Abschiedsformel, die Nähe zum Adressaten herstellen soll („Küsschen“).

(1) Hallo, könnte natürlich jetzt einfach nur auf lächeln senden klicken, vieleicht hab ich aber so eine kleine chance das ich mal von Dir höre, würde mich mächtig freuen, auf bald, liebe grüße, XYZ.

(2) ich finde dein profil interessant, wenn du magst, mail mir doch an XYZ. Bin kein Mitglied, kann nur eingeschränkt schreiben. ... LG und Küsschen XYZ

Dass in der Partnersuche ein nähesprachlicher Duktus vorherrscht, überrascht nicht; in Bezug auf Zeitungsanzeigen wurde dies in linguistischen Arbeiten auch schon nachgewiesen. So zeigt Daniela Schibli (2014: 16) in ihrer Untersuchung zu 187 Kontaktanzeigen aus dem Zürcher Tages-Anzeiger, dass die Anrede hier durchgängig in der 2. Person Singular erfolgt. ${ }^{9}$ Und auch Dominik Banhold (2013: 286), der Texte aus der Main Post ausgewertet hat, spricht von einer „starken Nähesprachlichkeit in Partnerschaftsanzeigen“.

Die Nähesprachlichkeit in (1) und (2) ist also als ein typisches Textsortenmerkmal im Kontext von Kontaktanzeigen. Dabei ist allerdings zu bedenken, dass es sich bei beiden Beispielen um Texte handelt, die an eine bestimmte Person gerichtet sind. Die Du-Anrede hat hier eine stärkere Signalwirkung als in einem Anzeigentext, der sich an eine imaginäre Person wendet. Dieser Umstand wird in den Arbeiten von Banhold (2013) und Schibli (2014) nicht diskutiert. Untersucht wurde hier nur, wie auch in älteren Publikationen zum Thema Partnersuche (vgl. z. B. Stolt 1976 und Riemann 1999), die Anzeigentexte selbst, nicht die daraus resultierende Anschlusskommunikation. ${ }^{10}$ Das hängt damit zusammen, dass diese Kommunikation nur schwer zugänglich ist; auch mir liegen nur wenige Beispiele eines Parship-Mitglieds vor. Dennoch wage ich zu behaupten, dass in vielen solchen Nachrichten nähesprachliche Merkmale auftreten - und dies, obwohl sich die Kommunikationspartner auf Parship nicht kennen. Auf Facebook dagegen kann man davon ausgehen, dass zwischen den Beteiligten,

8 Weitere Beispiele finden sich bei Marx (2012b). In ihren Textanalysen unterscheidet sie verschiedene „Emotionalisierungsstrategien“. Dazu gehören positive Lexeme wie süß oder lieb, emotionsausdrückende Interjektionen wie hachja oder Komplimente (vgl. Marx 2012b: 103-106). 9 Hier ein Beispiel aus ihrem Korpus: „Wo bist Du, auch einsamer Trucker, ab 42-j.?“. Vgl. auch den Titel der Arbeit von Birgit Stolt (1976): „Hier bin ich! - Wo bist Du? Heiratsanzeigen und ihr Echo“. Dieser Untersuchung liegen 551 Anzeigen (310 von Frauen, 241 von Männern) aus der Wochenzeitung Die ZEIT zugrunde.

10 Aus der Arbeit von Stolt (1976) ist aber immerhin zu entnehmen, dass die Zahl an Zuschriften, die auf eine Anzeige folgten, zwischen 0 und 196 liegt. 
die sich (z. B. im Chat) mit „Küsschen“ verabschieden, aller Wahrscheinlichkeit nach eine persönliche Bekanntschaft besteht.

Nähesprachlichkeit auf Facebook kann also (muss aber nicht) ein Reflex von Nähe sein; Nähesprachlichkeit auf Parship dagegen dient grundsätzlich dazu, Nähe herzustellen, ohne dass es zu Beginn eines Nachrichtenaustausches eine solche Nähe geben würde. Denn dies ist gerade das Prinzip der organisierten Partnersuche (ob im Internet, via Zeitung oder beim Speed-Dating): Die Beteiligten kennen sich nicht, zwischen ihnen bestehen keine „strong ties“ (vgl. dazu Granovetter 1973). ${ }^{11}$ Damit komme ich zu einem weiteren Unterschied zwischen Facebook und Parship: Auf Facebook kann es durchaus sein, dass die Akteure nicht die Absicht verfolgen, sich jemals offline zu treffen. Vielen genügt es, auf der Profilseite des anderen ,vorbeizuschauen', ab und zu etwas zu posten oder einfach nur zu lesen, was die anderen schreiben (vgl. dazu ausführlich Dürscheid/ Brommer 2013). Ziel auf Parship dagegen ist es, die Person, der man persönliche Nachrichten schickt, näher kennenzulernen und sie, sofern man Sympathie empfindet, möglichst bald persönlich zu treffen. Das persönliche Treffen hat hier einen großen Stellenwert, es steht gewissermaßen immer im Raum - und sei es, dass man es gerade deshalb noch eine Weile hinauszögert. Denn erst in einem persönlichen Gespräch zeigt sich, so formuliert es Döring (2009: o.S.), ob die Chemie zwischen zwei Menschen stimmt:

Durch Online-Kontaktbörsen lassen sich schnell, komfortabel und diskret Dutzende potenzieller Partner direkt kontaktieren. [...] Viele Teilnehmerinnen und Teilnehmer sind bei verschiedenen Portalen gleichzeitig angemeldet, pflegen parallel Kontakte zu diversen möglichen Dating-Partnern/-Partnerinnen und streben relativ schnell den Wechsel zu Telefonat und Treffen an, um festzustellen, ob die Chemie stimmt.

Wie Nicola Döring hier auch erwähnt, können Partnersuchende ihre Suche effizienter gestalten, wenn sie sich gleichzeitig bei verschiedenen Portalen anmelden und Parallelkontakte pflegen. Solch parallel stattfindende, bilaterale Kommunikationen sind aber auch auf ein und derselben Plattform möglich; auf Facebook können sie sogar quasi-synchron vollzogen werden (z. B. im Facebook-Chat). Auf Parship dagegen ist der Nachrichtenaustausch immer asynchron, ein Chatangebot gibt es nicht. Zudem vollzieht sich die bilaterale Kommunikation einzig mit

11 Worin die Unterschiede zwischen „strong“ und „weak ties“ liegen, wird hier nicht im Detail erläutert. Ich schließe mich Granovetter (1973: 1361) an, der schreibt: „It is sufficient for the present purpose that most of us can agree, on a rough intuitive basis, whether a given tie is strong, weak, or absent. “ Die Online-Partnersuche vollzieht sich im besten Falle in drei Schritten: absent $>$ weak > strong. 
„weak ties“, nicht mit „strong ties“. Es liegt also eine größere Unverbindlichkeit vor, was sich beispielsweise auch darin zeigt, dass man mehreren Personen gleichzeitig Avancen machen kann, aber auch jederzeit wieder umstandslos aus der Kommunikation aussteigen kann. Es genügt, die Nachricht des anderen unbeantwortet zu lassen. Dazu sei, den Punkt abschließend, Hans Geser zitiert, der in einem höchst lesenswerten Aufsatz mit dem Titel „Online Search for Offline Partners“ diese Kommunikationspraxis mit den folgenden Worten beschreibt:

Certainly, such poly-bilateral relationships facilitate self-disclosure because whenever I open myself up to partner A I have not to care that this same information will also be transmitted to partners B, C and D. On the other hand, they may remain perpetually unstable insofar as I can give them up easily without hurting anybody else beside my partner. (Geser 2007: 14)

\section{Die Courtship-Kommunikation}

Sucht man nach linguistischen Arbeiten zur Courtship-Kommunikation, dann stößt man rasch auf mehrere Publikationen aus einem DFG-Forschungsprojekt, das unter der Leitung von Joachim Knape stand. Die Ziele dieses Projekts, das am Seminar für Allgemeine Rhetorik der Universität Tübingen durchgeführt wurde, beschreiben Knape, Becker und Guhr (2009: 234) folgendermaßen (in Auszügen):

Es soll zum Ersten eine systematische Untersuchung des sprachlich-strategischen Flirtverhaltens erfolgen, diese sollte zum Zweiten unter rhetorischen Gesichtspunkten, also unter Konzentration auf den Sprecher in der Orator-Position erfolgen, der sein Ziel mit Hilfe von Strategien zu erreichen versucht. Als Drittes sollte erstmalig eine Betrachtung des Gesprächstyps „Erstkontaktgespräch“ erfolgen, der bislang in der deutschsprachigen wissenschaftlichen Literatur unbeachtet geblieben ist [...].

Wie im Text weiter ausgeführt, basierten die Untersuchungen in diesem Projekt auf einem Experiment. Die Probanden wurden darüber informiert, dass sie an einem Experiment teilnehmen, der Zweck wurde ihnen aber nicht mitgeteilt. Die Hälfte der Gruppe erhielt die Aufgabe, den Kommunikationspartner in einem Einzelgespräch innerhalb von 15 Minuten zum Austausch der Telefonnummern und einer weiteren Verabredung zu bewegen; die andere Hälfte sollte sich auf das Gespräch einlassen und - je nach Instruktion - positiv oder negativ auf die Annäherungsversuche reagieren. Die Gespräche wurden aufgezeichnet und ausgewertet; zudem sollten die Probanden im Nachhinein einen Fragebogen ausfüllen, in dem sie das Gespräch und die Gesprächspartner bewerteten (vgl. Knape/ Becker/Guhr 2009: 237). Im Zentrum der Untersuchung stand die Frage, welche 
persuasiven Strategien der Werbende (= der Orator) einsetzt, um die Sympathie einer anderen Person zu gewinnen und zu erreichen, dass es zu einer Anschlusskommunikation kommt. ${ }^{12}$ Dabei sollten nur die Handlungsweisen des Werbenden (des Orators) betrachtet werden, die Interaktion zwischen den Gesprächsbeteiligten stand nicht im Fokus.

Welche Parallelen gibt es nun zwischen dieser Art von Courtship-Kommunikation, die in einer Face-to-Face-Situation erfolgt, und der Suche nach einem Partner im Internet? Als Flirt kann man diese Kommunikation ja nicht bezeichnen, da zumindest zu Beginn eines solchen Kontakts keine bestimmte Person im Blickpunkt des Werbenden steht. Doch auch in der Online-Partnervermittlung ist der Werbende bemüht, sich positiv darzustellen, und auch hier verwendet er verschiedene Strategien, um das Interesse eines potenziellen Partners zu wecken. Da es zunächst aber nur um einen imaginären Partner geht, den er auf der Profilseite umwerben kann, und da zu diesem Zeitpunkt noch keine Interaktion stattfindet, fallen Handlungsphasen weg, die in einem „Erstkontaktgespräch“ von Bedeutung sind. Diese sind, wie in einer weiteren Arbeit aus dem CourtshipProjekt dargelegt wird, „das Kennenlernen (bzw. Informationen akquirieren), das Gemeinsamkeiten finden (bzw. Herstellen eines gemeinsamen Bezugssystems), das Sympathie gewinnen (bzw. Attraktion generieren durch positive Selbstdarstellung) und das Sympathie prüfen“ (Becker 2009: 268). Natürlich kann der Schreiber auf einer Profilseite versuchen, Gemeinsamkeiten mit dem Leser herzustellen (vgl. z. B. das obige Zitat „Du spürst, was ich denk!!“), da sich diese Worte aber an eine imaginäre Person richten, sind sie nicht mit der CourtshipKommunikation in einer Face-to-Face-Situation vergleichbar. Das gilt auch für die Handlungsphase, die Becker als „Sympathie prüfen“ bezeichnet. Es gibt zu diesem Zeitpunkt ja noch keine Person, in Bezug auf die man überprüfen könnte, ob sich Sympathie zeigt. Daher steht in der Online-Partnersuche zunächst nur die dritte Phase, das „Sympathie gewinnen“, im Zentrum des Geschehens: Der Schreiber versucht, ein positives Selbstbild von sich aufzubauen und so den Leser für sich zu gewinnen. ${ }^{13}$

Das Gesagte trifft auch auf Partnerschaftsanzeigen in Zeitungen zu, allerdings weisen solche Annoncen im Vergleich zu den Profilseiten im Internet einen großen Nachteil auf: Der Schreiber hat nur wenige Zeilen zur Verfügung, um den

12 In gewisser Hinsicht ist dies vergleichbar mit Bewerbungs- und Verkaufsgesprächen; auch hier bietet „der Werbende sich selbst oder seine Produkte dem Umworbenen an“ (Guhr 2008: 30). 13 Gelingt ihm dies, kommt es zu einem Nachrichtenaustausch - und dann (erst) werden auch die anderen Handlungsphasen relevant (z. B. Informationen akquirieren, Herstellen eines gemeinsamen Bezugssystems). 
Leser von der eigenen Person zu überzeugen. In diesen wenigen Zeilen äußert er sich, wie Banhold (2013: 280) schreibt, in der Regel nicht nur zur eigenen Person (z. B. ich, 33, schlank), sondern macht auch Angaben zum Wunschpartner (z. B. möglichst im Raum Düsseldorf). Interessanterweise sieht Parship diese Möglichkeit an keiner Stelle explizit vor, die Selbstdarstellung steht auf der Website im Vordergrund. ${ }^{14}$ Hierfür stellt das System mehrere Optionen zur Verfügung. So soll man allgemeine Informationen zur eigenen Person geben (z. B. Schulabschluss, Raucher/Nichtraucher, Körpergröße, Zahl der Kinder, Heimatregion), man kann sich aber auch über eine Auswahl vorgegebener Attribute charakterisieren (z. B. „attraktiv, sehr attraktiv, lebenslustig, erfolgreich, selbstbewusst, liebevoll, zielstrebig, reiselustig, zuverlässig, weltoffen“). Weiter wird man beim Erstellen der Profilseite dazu aufgefordert, vom System vorgeschlagene Textbausteine zu Ende zu führen und auf diese Weise sein Profil „noch persönlicher zu machen“: ${ }^{15}$

Ich über mich Freizeit \& Sport Fotoalbum

Beantworten Sie weitere Fragen, um Ihr Profil noch persönlicher zu machen. So erhöhen Sie Ihre Chancen auf mehr Kontaktanfragen.

Ein Tag ist für mich perfekt, wenn...

ich alles gemacht habe، was ich mir vorgenommen habe, und es auch etwas Schönes gab

Abb. 3: Gestaltung einer Profilseite auf Parship.

Solche Informationen können dem Leser als Anhaltspunkte in seinem Selektionsprozess dienen, das oben erläuterte Gatekeeping basiert im Wesentlichen darauf. Das gilt auch für Bilder, die in der Rubrik „Fotoalbum“ hochgeladen werden. Allerdings sind diese bei Parship in der Regel nicht sofort einsehbar, sie

14 Das lässt sich damit begründen, dass zu Beginn der Mitgliedschaft ein 70 Fragen umfassender Test bearbeitet werden soll, der dazu dient, die „Partnerschaftspersönlichkeit“ (so die Formulierung von Parship) zu ermitteln. Zudem muss jedes neue Mitglied Angaben zum Wunschpartner machen (z. B. Alter, Körpergröße und Region). Diese Angaben erscheinen nicht als Text auf der Profilseite, sie dienen dem System aber als Selektionskriterium bei der Zusammenstellung von Partnervorschlägen.

15 Auf ElitePartner wird diesbezüglich anders verfahren; hier soll jedes Mitglied selbst einen längeren Text formulieren. Das ist für den Schreiber zweifellos anspruchsvoller, für den Leser aber aufschlussreicher. 
müssen zunächst freigegeben werden. Insofern ist es zunächst einzig der Text (und nicht das Bild), der dem Partnersuchenden als Entscheidungsgrundlage dienen kann. In einer Face-to-Face-Interaktion hat er dagegen mehr Anhaltspunkte für das Gatekeeping (u. a. Äußeres, Mimik, Gestik, Ausstrahlung, Kleidung des Gegenübers), außerdem können die Gesprächspartner hier unmittelbar aufeinander reagieren. Ein weiterer Unterschied zur prototypischen CourtshipKommunikation besteht darin, dass der Fokus zunächst nicht auf eine bestimmte Person gerichtet ist. So wendet man sich auf der Profilseite einer Online-Partnervermittlung an alle Interessenten. Andererseits tut man dies oft auf eine Weise, als habe man nur eine Person im Blick. Das zeigen Formulierungen, mit denen der Leser direkt angesprochen werden soll (vgl. Abb. 4). Im folgenden Beispiel wird dem Leser gar dieselbe Bedeutung wie den eigenen Kindern beigemessen. Solche Formulierungen sind auf den ersten Blick irritierend. Doch kann es aus Sicht des Schreibers ja durchaus sein, dass für ihn der Wunschpartner so wichtig ist wie die eigenen Kinder.

Drei Dinge, die mir wichtig sind:

Nicht Dinge, Menschen: meine zwei Kinder - und du ;-)

Diese Person würde ich gerne einmal treffen:

Dich - aber nicht nur einmal :-)

Abb. 4: Adressatenbezug auf einer Profilseite.

\section{Textgestaltung in der Online-Partnersuche}

Im vorangehenden Abschnitt wurde gezeigt, dass die Online-Partnersuche eine Variante der Courtship-Kommunikation darstellt, dass es aber auch wesentliche Unterschiede zum prototypischen Courtship gibt. Das betrifft zum einen die Rahmenbedingungen (Face-to-Face/gesprochen vs. Screen-to-Screen/geschrieben), zum anderen den Umstand, dass es in einem Fall um eine einseitige Annäherung geht, im anderen Fall um eine wechselseitige. Beiden Courtship-Varianten gemeinsam ist, dass die Sprache in diesem Annäherungsprozess eine wichtige Rolle spielt. Der Schweizer Anglist Ernst Leisi hat dem Thema „Paar und Sprache“ denn auch ein ganzes Buch gewidmet, das inzwischen bereits in der vierten Auflage erschienen ist. Er beginnt sein Buch mit den folgenden Worten: 
Die Paarbeziehung gehört ohne Zweifel zu den wichtigsten Beziehungen, deren der Mensch fähig ist. [...] Innerhalb dieser Beziehung spielt die Sprache eine sehr wichtige Rolle. Ein dauernd schweigendes Paar ist bei normal Sprachbegabten nicht denkbar. Die Beziehung wird weitgehend durch Sprache angebahnt; an ihrem weiteren Verlauf ist die Sprache stets beteiligt. Ob die Beziehung fortdauert oder zusammenbricht, kann von sprachlichen Dingen abhängen. (Leisi 1993: 7, Hervorhebung im Original)

In den fünf Hauptkapiteln seines Buches unterscheidet Leisi nicht zwischen den beiden Modalitäten von Sprache (gesprochen/geschrieben); ihm geht es generell - so der Untertitel - um die linguistischen Aspekte der Zweierbeziehung. Dies ist im vorliegenden Aufsatz anders; hier stehen modalitätsspezifische Fragen im Zentrum (vgl. zu dieser Perspektive auch Wyss 2011): Welche Möglichkeiten hat eine Person, wenn die Beziehungsanbahnung nur schriftlich erfolgen kann? Wie nutzt sie die Profilseite, um für sich zu werben und - um im Bild zu bleiben - im ersten Gatekeeping durch das Tor zu gelangen? Liegt nicht die Vermutung nahe, dass sie im Schreiben besonders viel Mühe aufwenden wird, um sich in ein positives Licht zu rücken? Um Antworten auf diese Fragen zu erhalten, seien die wenigen Arbeiten konsultiert, die das Thema Online-Partnersuche aus linguistischer Sicht behandeln. Dazu gehört ein Aufsatz von Andrea Bachmann-Stein, der den Titel „Kontaktanzeigen im World Wide Web“ trägt und in dem die These vertreten wird, dass sich „zwischen Print-Kontaktanzeigen und Online-Kontaktanzeigen [...] auf funktionaler und inhaltlich-thematischer Ebene keine bzw. kaum Unterschiede finden“ (Bachmann-Stein 2011: 109). Das allerdings muss noch genauer untersucht werden, denn Profilseiten in der Online-Partnervermittlung bieten weitaus mehr Möglichkeiten der Selbstdarstellung als Zeitungsannoncen. ${ }^{16}$ Bachmann-Stein stellt weiter fest, dass in den von ihr untersuchten OnlineAnzeigen zahlreiche „Verstöße[n] gegen sprachliche Normen“ auftreten würden. Sie erläutert dies an einem Beispiel, das hier im Zitat wiedergegeben wird:

Es finden sich zwar auch Texte, die normgemäß realisiert werden, sie stellen im Korpus jedoch die Minderheit dar. Der Bereich, in dem sich die meisten Fehler finden, ist - wenig überraschend - die Orthographie, aber auch die Syntax ist davon betroffen (vor allem Akkusativergänzungen werden zum Teil wie Nominativergänzungen realisiert, z. B. [...] Ich Möchte nen Richtigen Kerl - und kein schleimenden, sensiblen Hetero Schwulen. (BachmannStein 2011: 105)

Dass es zu solchen Normverstößen kommt, begründet Bachmann-Stein damit, dass im Internet einerseits eine größere Toleranz gegenüber normabweichender

16 Insofern ist es aus meiner Sicht nicht passend, diese als „Online-Kontaktanzeigen“ zu bezeichnen. Es handelt sich vielmehr um eine neue Textsorte, die ihre eigenen Merkmale aufweist. 
Schreibung herrsche, andererseits aber auch Fehler von den Schreibern schneller übersehen würden. Das von ihr zitierte, etwas befremdende Beispiel stammt von der Internetplattform „rubensfan.de“, einer „Singlebörse für mollige und dicke Singles“ (Zitat von der Website). Dabei handelt es sich um ein kostenloses Angebot, das weitaus weniger Möglichkeiten als die kostenpflichtigen Plattformen von Parship oder ElitePartner bietet. Die Vermutung liegt nahe, dass derjenige, der ein kostenpflichtiges Angebot nutzt, sich mehr Mühe in der Gestaltung der Profilseite geben würde. Doch das ist nur eine Vermutung; es wäre interessant, in dieser Hinsicht kostenpflichtige Online-Partnervermittlungen mit kostenlosen Angeboten zu vergleichen. Auch würde es sich lohnen, auf empirischer Grundlage der grundsätzlichen Frage nachzugehen, welchen Stellenwert die schriftlichen Ausdrucksfähigkeiten in der Online-Partnervermittlung haben. Und dabei sollte nicht nur der Schreibstil untersucht werden, der zweifellos eine wichtige Rolle spielt (z. B. Originalität, Sprachwitz), sondern auch die formale Seite der Texte. Denn kann es nicht sein, dass - anders als Andrea BachmannStein es vermutet - die Schreiber auf solchen Plattformen nachgerade bestrebt sind, Rechtschreib- und Grammatikfehler zu vermeiden? Im Folgenden möchte ich diese Vermutung auf theoretischer Ebene begründen. Dazu beziehe ich mich auf Überlegungen des Philosophie-Professors Aaaron Ben-Ze'ev (2004) von der Universität Haifa. In seinem Buch „Love Online. Emotions on the Internet“ erläutert er, warum man in der Online-Welt dazu tendiere, mehr von sich preiszugeben als in der Offline-Welt:

The greater tendencies toward self-disclosure in cyberspace can also be explained by a lack of the usual "gating features" - easily discernible features such as unattractive external appearance, stereotypic characteristics, visible shyness, or social anxiety - which might be an obstacle to the establishment of any close relationship. (Ben-Ze'ev 2004: 37-38)

Diese Aussage findet sich in einem Kapitel mit der Überschrift „Lack of gating features“. ${ }^{17}$ In dem Kapitel macht Ben-Ze'ev (2004: 38) einen Unterschied zwischen „external gating features“ und „internal gating features“. Erstere seien solche, die jemanden von einem Annäherungsversuch abhalten könnten (z. B. das Aussehen des anderen), Letztere solche, welche die betreffende Person selbst von einer Kontaktaufnahme abhalten würden (z. B. die eigene Schüchternheit). Im Internet würden, so Ben-Ze'ev, solche „gating features“ an Bedeutung verlie-

17 Ben-Ze’ev spricht von „gating features“, nicht von „gatekeeping features“. Ich fasse dies hier als Quasi-Synonyme auf. Im einen Fall steht der Torwächter im Zentrum der Metaphorik, im anderen Fall das Tor. 
ren, der Cyberspace mache es möglich, sich näher zu kommen, „without having to deal directly with such gates“ (Ben-Ze'ev 2004: 38).

So zutreffend diese Bemerkungen sind, einen Punkt lässt der Autor in diesem Kapitel außer Acht: Auch in der Online-Welt gibt es „gating features“, nur sind diese von anderer Art als in der Offline-Welt. $\mathrm{Zu}$ den „external gating features“ im Internet zählen beispielsweise die Ausdrucksmöglichkeiten, über die eine Person verfügt. Ben-Ze'ev (2004: 166) selbst deutet dies an späterer Stelle an, wenn er schreibt: „In cyberlove [...] verbal skills are more important than physical attractiveness“. ${ }^{18}$ Das führt mich zu der These, dass in der schriftbasierten Courtship-Kommunikation das Schreiben (und damit auch die Orthographie) eine zentrale Funktion einnimmt. Mit anderen Worten: Der Leser entscheidet auf der Basis des Geschriebenen, ob der Schreiber für ihn interessant sein könnte oder nicht. Auch Hans Geser, der in seinen Ausführungen auf Ben-Ze'ev (2004) Bezug nimmt, argumentiert in diese Richtung. Er geht sogar so weit zu sagen, dass Rechtschreib- und Grammatikfehler vom Leser als Indikatoren für mangelnde Schulbildung (oder gar Intelligenz) angesehen werden könnten:

In particular, writing skills may be taken as a valid proxy for personal intelligence, charm and creativeness and as a signal for more generalized social and communicative skills. For instance, highly salient features like mistakes in orthography and grammar may become straightforward indicators of deficient individual schooling (or even intelligence), and be used as potent screening criteria on the competitive partner matching markets. (Geser 2007: 17)

Stimmt man dieser Aussage zu, dann kann man noch einen Schritt weiter gehen und daraus folgern, dass der Schreiber im Selektionsprozess nur dann eine Chance erhält, wenn sein Text wenig Rechtschreib- und Grammatikfehler aufweist. Im Umkehrschluss heißt das aber auch, dass derjenige, der für sich werben möchte, ein Interesse daran haben wird, den Text auf seiner Profilseite möglichst fehlerfrei zu gestalten. Denn (auch) auf diese Weise kann er beim Leser den Eindruck erwecken, gebildet zu sein; zudem kann ein sorgfältig verfasster Text dem Leser signalisieren, dass es dem Schreiber ernst mit der Partnersuche ist. Hinter solchen - möglicherweise falschen - Rückschlüssen steht der Halo-Effekt, mit dem in der Psychologie das Phänomen bezeichnet wird, dass von bestimmten Eigenschaften einer Person (z. B. ihrem sozialen Status) auf andere (z. B. ihre Intelligenz) geschlossen wird. In Bezug auf die Online-Partnersuche heißt das: Nicht nur der Inhalt des Textes, sondern auch die Form (ver-)führt den Leser

18 Einem Kapitel seines Buches stellt er gar das folgende Motto voran: „All really great lovers are articulate, and verbal seduction is the surest road to actual seduction“ (Ben-Ze'ev 2005: 145). 
dazu, Rückschlüsse auf die Person des Schreibers zu ziehen. ${ }^{19}$ Hierzu sei nochmals Aaron Ben-Ze'ev zitiert:

A common phenomenon in offline relationships is the „attractiveness halo“, in which a person who is perceived as beautiful is assumed to have other good characteristics as well. In online relationships, this is replaced by the "personality halo", in which a person who is perceived as having a specific, positive personality trait is assumed to have other good characteristics - sometimes even those connected to external appearance. (Ben-Ze'ev 2004: 161)

Wer also jemandem schriftlich den Hof machen möchte, der sollte, zugespitzt gesagt, auf die Orthographie und Grammatik seiner Texte achten, denn diese machen einen Teil des „personality halo“ aus. In gewisser Weise sind Fehler in diesem Bereich, wiederum zugespitzt gesagt, vergleichbar mit einer unordentlichen Kleidung oder einer schlecht sitzenden Frisur (,attractiveness halo“). Dabei macht es natürlich einen Unterschied, ob man die betreffende Person schon kennt und ihr „Flirtbriefe“ schickt (vgl. Wyss 2011) oder ob man online auf Partnersuche ist und seine Profilseite gestaltet. Zudem mag man einwenden, dass im Internet ohnehin eine größere Normentoleranz vorherrscht und Orthographie- und Grammatikfehler deshalb nicht so sehr ins Gewicht fallen. In diese Richtung gehen ja auch die Überlegungen von Andrea Bachmann-Stein (s. o.). Allerdings muss man dabei den jeweiligen Handlungsrahmen berücksichtigen: Wer auf Facebook chattet, der kann vielleicht von einer solchen Normentoleranz ausgehen (v.a. auch dann, wenn er den anderen persönlich kennt), wer dagegen ein Profil auf Parship anlegt, der muss sich möglichst gut präsentieren - und dazu gehört auch die formale Seite des Textes.

Doch sind das nur Vermutungen; es könnte - ganz im Gegenteil - ja auch sein, dass normfernes Schreiben als Zeichen von Lockerheit angesehen wird und gerade deshalb sympathisch wirkt. Wirft man aber einen Blick in das Diskussionsforum von Parship (vgl. https://www.parship.ch/forum/), dann gewinnt man den Eindruck, dass das korrekte Schreiben in der Online-Partnersuche tatsächlich eine nicht unwichtige Rolle spielt. In diesem Forum besteht die Möglichkeit, Fragen zu stellen (z. B. zum Thema „Dating-Tipps“, „Beziehung“ oder „Profilbe-

19 In einer E-Mail an mich schreibt Hans Geser dazu, dass „Kommunikationspartner [...] eine starke Tendenz haben, faute de mieux aus allen verfügbaren digitalen Cues oft recht spekulative Schlüsse auf die Persönlichkeit, das Denken, Fühlen und die Absichten des Senders zu ziehen, wobei vor allem auch metakommunikative Merkmale (z. B. Umfang und Timing von Reaktionen, ja sogar die Tageszeit ihrer Abfassung) von Bedeutung sind. "Was den Nachrichtenaustausch auf Parship betrifft (s. u.), ist das sicher der Fall: Wartet man zu lange mit einer Antwort oder fällt die Nachricht sehr kurz aus, muss man damit rechnen, dass dies vom anderen als Desinteresse ausgelegt wird. 
ratung“), die von anderen Mitgliedern oder dem Parship-Administrator beantwortet werden. Einige Beispiele sollen hier präsentiert werden, um deutlich zu machen, dass die oben geäußerten Vermutungen ihre Berechtigung haben. ${ }^{20}$ So zeigt der folgende Dialog (in Originalschreibung) an, welche Tipps auf die Frage gegeben werden, wie man das eigene Profil attraktiver gestalten könnte:

(A) Ich würde euch bitten mein Profil zu bewerten, leider lassen Kontakte sehr auf sich warten oder melden sich so gut wie überhaupt nicht bzw. schreiben in den seltensten Fällen zurück.

(B) bitte noch mal Großschreibung (die Ruhe, mein Ich, das Wichtigste) und Interpunktion (es fehlt hier und da ein Komma ...) prüfen.:

(A) Ich habe mich über die Jahre daran gewöhnt im Internet meistens nicht auf richtige Punktierung bzw. Groß- und Kleinschreibung zu achten. Dies scheint mir offensichtlich (dank deiner Analyse) ein wenig zum Verhängnis geworden zu sein.

(A) erhält noch eine ganze Reihe weiterer Ratschläge zur Gestaltung seiner Profilseite (z. B. besseres Foto, geschicktere Wortwahl). Doch darauf kommt es hier nicht an. Wichtig für den vorliegenden Zusammenhang ist, dass darunter auch die Orthographie fällt (hier die Großschreibung und die Interpunktion). Dass diese in der Profilgestaltung einen wichtigen Stellenwert hat und von vielen Lesern kritisch geprüft wird, zeigt auch der folgende kleine Test: Gibt man im Diskussionsforum als Suchbegriff z. B. das Stichwort, Rechtschreibung‘ ein, dann erhält man 485 Treffer (z. B. „Mir fällt immer wieder auf, dass die meisten Männer hier der deutschen Sprache nicht wirklich mächtig sind. Entweder sind sie gar nicht in der Lage, mehrere Sätze vernünftig zu formulieren oder es wimmelt vor lauter Rechtschreib- und Zeichensetzungsfehlern.“). Sicher sind unter diesen vielen Treffern auch einige, die für die vorliegende Argumentation nicht relevant sind. Doch zeigt die große Zahl eine Tendenz auf, der man nachgehen sollte. ${ }^{21}$

Neben aller Originalität im Ausdruck und einem guten Schreibstil spielt also in der Online-Partnersuche die formale Gestaltung der Texte, dies meine These, ebenfalls eine wichtige Rolle. $\mathrm{Zu}$ dieser Annahme gelangt auch Georg Albert, der für seine Dissertation zum Thema „Innovative Schriftlichkeit in digitalen Texten“ eine Stichprobe aus dem Diskussionsforum von ElitePartner ausgewertet hat. Auch in diesem Forum besteht die Möglichkeit, bei den Mitgliedern

20 Doch auch dadurch lassen sich diese Vermutungen nicht empirisch abstützen; dazu bräuchte es eine umfassende Befragung von Parship-Mitgliedern.

21 Zum Vergleich: Zum Stichwort ,Grammatik‘ erhält man 129 Treffer (Stand: 22. 9. 2016). Hier ein Beispiel: „Grundsätzlich ist Dein Profil angenehm und sympathisch; Rechtschreibung und Grammatik sind korrekt und lassen niemanden einen inneren Schreikrampf kriegen.“ Interessant wäre in diesem Zusammenhang auch, der Frage nachzugehen, ob Orthographie- und Grammatikfehler eher von Männern oder (was ich vermute) von Frauen angemahnt werden. 
Verbesserungsvorschläge zur Gestaltung der eigenen Profilseite einzuholen. Albert stellt dazu fest: „Die Überarbeitung der formal-sprachlichen Gestaltung wird dabei immer wieder angemahnt und zum entscheidenden Kriterium der Fremdwahrnehmung erklärt“ (Albert 2013: 153). Die Normorientierung erklärt er damit, dass sich die Mitglieder auf diese Weise von Nutzern anderer Plattformen abgrenzen wollen, deren Angebote sie als „unseriös“ und „niveaulos“ (Originalzitate von Forumsmitgliedern) bewerten würden. Albert stellt dazu abschließend fest: „Sichtbares Zeichen dieser Distinktion - und Herausstellung eines eigenes „Niveaus“ - ist [...] die formale Korrektheit der selbstverfassten Texte, inklusive standardkonformer Orthographie“ (Albert 2013: 153).

Dass der Anspruch von ElitePartner, eine Plattform für „Akademiker \& Singles mit Niveau“ zu sein, tatsächlich ein Grund für die hohe Wertschätzung der Rechtschreibung ist, mag sein. Doch wie wir weiter oben gesehen haben, gibt es auch auf Parship Kommentare, die auf die formale Gestaltung der Profilseite abzielen. Offensichtlich scheint eine korrekte Rechtschreibung auch für viele Parship-Mitglieder einen hohen Stellenwert zu besitzen. Das zeigt auch der folgende Beitrag, mit dem der Parship-Administrator (vgl. A) im Forum eine Diskussion zu der Frage lanciert, welchen Status die Rechtschreibung für die Mitglieder hat. ${ }^{22}$ Auf seine Frage, die im Folgenden im Original wiedergegeben ist (und selbst zwei Interpunktionsfehler enthält), folgen 106 Beiträge. Drei dieser Antworttexte (ebenfalls in Originalschreibung) werden in Auszügen zitiert (vgl. B bis D):

(A) „Mahgst du Rockmusig auch so sähr wie ich?“ - Wie wichtig ist Ihnen korrekte Rechtschreibung im Profil und in den Nachrichten. Kommt ein Partner mit Rechtschreibschwierigkeiten überhaupt in Frage?

(B) Für mich ist der korrekte Gebrauch unserer schönen Sprache ein absolutes Muss. Wenn ich eine Nachricht bekomme, die grobe Fehler enthält, stehen die Chancen auf eine Antwort von mir eher schlecht. Rechtschreibung und Grammatik sind erste Anhaltspunkte für die Bildung des Absenders. Und darauf lege ich schon wert.

(C) Naja. Ich finde, es kann auch mal nur ne schnelle Antwort sein - wenn da der Satzbau oder die Rechtschreibung nicht $100 \%$ Duden-Niveau haben, ist das für mich nicht so schlimm. Kommt auch drauf an, ob das jetzt die erste Nachricht ist, oder die 5. die man sich schreibt.

(D) Ich achte eigentlich bei jedem Partnervorschlag auf die richtige Rechtschreibung. Tippfehler in einem Text zeigen mir, dass die Person sich gar keine Mühe gemacht hat und die Nachricht einfach schnell abschicken wollte. Wenn aber richtige Rechtschreibfehler auftreten, finde ich es ja sogar noch schlimmer: das bissschen Bildung muss doch schon vorausgesetzt sein!

22 Siehe unter https://www.parship.ch/forum/single-leben/korrekt-schreiben-ein-muss-87. html [22.9. 2016]. 
Wie die Antwort von (C) zeigt, macht dieser einen Unterschied zwischen der Textgestaltung auf der Profilseite und den persönlichen Nachrichten, die auf Parship verschickt werden. Bei Letzteren scheint seine Toleranz gegenüber Normverstößen größer zu sein - zumindest, so hält er fest, wenn es nicht die erste Nachricht ist, die man sich schreibt. Das würde bestätigen, was in Arbeiten zur computervermittelten Kommunikation betont wird: Je schneller der „Interaktionsrhythmus“ (vgl. Androutsopoulos 2007: 89) ist, desto informeller werden die Texte und desto eher akzeptiert man als Leser die Informalität solcher Texte (und die darin auftretenden Flüchtigkeitsfehler). Doch das beschreibt nur eine Tendenz; es muss immer auch der Handlungskontext berücksichtigt werden. In der OnlinePartnersuche will man sich ein Bild von der anderen Person machen - und dafür hat man als Anhaltspunkt nur das, was auf dem Bildschirm erscheint. Deshalb gilt auch für diese zweite Phase des Gatekeepings: Wem es nicht nur auf der Profilseite, sondern auch im Schreiben seiner persönlichen Nachrichten gelingt, sich angemessen auszudrücken und Schreibfehler auf ein Minimum zu reduzieren, der hat gute Chancen, im Selektionsprozess einen Schritt weiterzukommen.

\section{Ausblick}

Meines Wissens gibt es keine linguistische Arbeit, die sich auf breiter empirischer Grundlage mit der Beziehungsanbahnung im Netz befassen würde. Die meisten der $\mathrm{zu}$ diesem Thema vorliegenden Untersuchungen haben entweder einen medienpsychologischen oder einen soziologischen Zugang (so auch Skopek et al. 2009), oder sie fokussieren einen Aspekt, in dem nicht die Partnersuche selbst im Zentrum steht. So wertet Georg Albert zwar eine Reihe metasprachlicher Kommentare im Diskussionsforum von ElitePartner aus, es geht ihm aber nicht um die Frage, wie die Online-Partnersuche aus linguistischer Sicht zu beschreiben ist. Andrea Bachmann-Steins Beitrag setzt zwar an dieser Stelle an, doch ihre Beobachtungen basieren, wie auch die Ausführungen von Konstanze Marx, auf einer sehr heterogenen Datengrundlage. So heißt es in dem Beitrag von BachmannStein vage, dass der Arbeit eine Sammlung von „mehreren Hundert Kontaktanzeigen“ zugrunde liegt, die sowohl aus Zeitungen stammen als auch von den „Online-Portale[n] yahoo.de, parship.de und verschiedener kostenlos zugänglicher Spezialportale“ (Bachmann-Stein 2011: 96); offensichtlich wurde hier also die Partnersuche via Zeitung und im Internet zusammengefasst. Und Konstanze Marx schreibt in einer Fußnote lediglich, dass ihr Korpus aus „ca. 1000 Emails, Chatsequenzen und Kontaktbörsen-Postings“ (Marx 2012b: 97) bestehe. Als „Emails“ [sic] bezeichnet sie vermutlich die persönlichen Nachrichten, die über 
die Website von Online-Partnervermittlungen verschickt werden. Was in diesem Kontext aber unter „Chatsequenzen“ zu verstehen ist, bleibt unklar.

Diese Forschungslücke in der Empirie kann auch der vorliegende Beitrag nicht schließen; um eine datenbasierte Untersuchung handelt es sich hier nicht. Mein Ziel war ein anderes: Wichtig war mir, die Aufmerksamkeit des Lesers auf ein Thema zu lenken, das in dem weiten Feld von Sprache und Beziehung eine wichtige Rolle spielt, und aufzuzeigen, welche Aspekte aus linguistischer Sicht relevant sein können, wenn man die kommunikativen Aktivitäten in der OnlinePartnersuche genauer untersuchen möchte. So habe ich die Frage diskutiert, auf welchen äußerlichen Merkmalen der Halo-Effekt basiert, wenn die CourtshipKommunikation nur schriftlich erfolgt. Weiter wurde in den vorangehenden Abschnitten auf die Gatekeeping-Funktion des Textes verwiesen und die These vertreten, dass in der Online-Partnersuche die formale Gestaltung (und hier insbesondere die Orthographie) eine wichtigere Rolle spielt, als man zunächst vermuten könnte. Dabei stellte sich aber auch die Frage, ob diese Aussage nur für die Profilseiten zutrifft und nicht auch für den Austausch persönlicher Nachrichten, also für die Phase, in der das Gatekeeping seine Fortsetzung findet. Doch wird es schwer sein, Einblicke in solche Nachrichten zu bekommen, denn sie betreffen einen sehr privaten Bereich. Hätte man Zugang zu diesen Daten, dann könnte man auch „nicht-geglückte Erstkontaktaufnahmeversuche“ (Marx 2012b: 106) untersuchen, also solche, in denen der Nachrichtenwechsel nach kurzer Zeit wieder eingestellt wurde.

Um herauszufinden, welche Gründe in der Online-Partnersuche zu einem Abbruch des Kontakts führen, sollte man aber nicht nur die Texte selbst analysieren, sondern auch Befragungen durchführen. Personen, die bereits seit einiger Zeit Mitglied in einer Online-Partnervermittlung sind und schon viele Erstkontakte geknüpft, aber auch wieder beendet haben, würden sich hierfür besonders eignen. Solche Befragungen können einen weiteren Hinweis darauf geben, ob die schriftlichen Ausdrucksfähigkeiten in der Online-Partnersuche tatsächlich eine Gatekeeping-Funktion haben. Doch auch wenn sich diese Annahme bestätigen sollte (und davon gehe ich aus): Es sind andere Kriterien, die - selbst wenn man in der Online-Welt durch das Tor gelangt ist - den Ausschlag dafür geben, ob es in der Offline-Welt tatsächlich zu einer Liebesbeziehung kommt. Hierzu sei abschließend ein Teilnehmer aus dem ElitePartner-Forum zitiert (Zitat übernommen von Albert 2013: 154): ,also ich habe mich noch nie verliebt, weil ihr deutsch so gut war." 


\section{Literatur}

Albert, Georg (2013): Innovative Schriftlichkeit in digitalen Texten. Syntaktische Variation und stilistische Differenzierung in Chat und Forum. Berlin.

Androutsopoulos, Jannis (2007): Neue Medien - neue Schriftlichkeit? In: Mitteilungen des Deutschen Germanistenverbandes 1/07, 72-97.

Bachmann-Stein, Andrea (2011): Kontaktanzeigen im World Wide Web. In: Patrick Schäfer/ Christine Schowalter (Hg.): In mediam linguam. Mediensprache - Redewendungen Sprachvermittlung. Festschrift für Heinz-Helmut Lüger. Landau, 95-110.

Banhold, Dominik (2013): Bitte melde dich! Syntaktisch-pragmatischer Standard in Partnerschaftsanzeigen. In: Jürg Hagemann/Wolf Peter Klein/Sven Staffeldt (Hg.): Pragmatischer Standard. Tübingen, 277-289.

Beck, Klaus (2013): Gatekeeper. In: Günter Bentele/Hans-Bernd Brosius/Otfried Jarren (Hg.): Lexikon Kommunikations- und Medienwissenschaft. 2., überarbeitete und erweiterte Auflage. Wiesbaden, 100.

Becker, Nils (2009): Zum Problem der Struktur und Steuerung erotischer Partnerwerbungsgespräche. In: Joachim Knape (Hg.): Rhetorik im Gespräch. Ergänzt um Beiträge zum Tübinger Courtshiprhetorik-Projekt. Berlin, 251-294.

Ben-Ze'ev, Aaron (2004): Love online. Emotions on the internet. Cambridge.

Döring, Nicola (2009): Liebe per Mausklick? Chancen und Risiken der Partnerwahl im Internet. In: BzgA Forum Sexualaufklärung und Familienplanung 2 (Partnerschaft). Online unter: http://forum.sexualaufklaerung.de/index.php?docid=1239 [21.9. 2016].

Dürscheid, Christa/Sarah Brommer (2013): Ist ein Freund noch ein Freund? Facebook und Sprachwandel. In: Der Deutschunterricht 2/2013 (Themenheft: Sprache und Generation), 28-40.

Frick, Karina (2014): Liebeskommunikation über Facebook. Eine korpusbasierte Untersuchung kommunikationstheoretischer und sprachlicher Merkmale der Paar-Kommunikation auf Facebook. Hannover (= Networx 65).

Online unter: http://www.mediensprache.net/networx/networx-65.pdf [20.9. 2016].

Geser, Hans (2007): Online search for offline partners. Matching platforms as tools of empowerment and retraditionalization. In: Sociology in switzerland: Towards cybersociety and vireal social relations. Zürich.

Online unter: http://socio.ch/intcom/t_hgeser19.pdf [22.9. 2016].

Granovetter, Mark (1973): The strength of weak ties. In: American journal of sociology 78, $1360-1380$.

Guhr, Dagny (2008): Argumentation in Courtshipkommunikation. Zu den persuasiven Strategien im Gespräch. Berlin.

Knape, Joachim (Hg.) (2009): Rhetorik im Gespräch. Ergänzt um Beiträge zum Tübinger Courtshiprhetorik-Projekt. Berlin.

Knape, Joachim/Nils Becker/Dagny Guhr (2009): Das Tübinger Projekt zur Courtshiprhetorik. In: Joachim Knape (Hg.): Rhetorik im Gespräch. Ergänzt um Beiträge zum Tübinger Courtshiprhetorik-Projekt. Berlin, 233-249.

Kwon, K. Hazel et al. (2012): Audience gatekeeping in the twitter service: An investigation of tweets about the 2009 gaza conflict. In: AIS transactions on human-computer interaction 4/4, 212-229. 
Leisi, Ernst (1993): Paar und Sprache. Linguistische Aspekte der Zweierbeziehung.

4., durchgesehene Auflage [1. Auflage 1978]. Heidelberg/Wiesbaden.

Marx, Konstanze (2012a): „XYZ hat dich angestupst“ - Romantische Erstkontakte bei Facebook - Ein Schnittstellenphänomen? In: Christina Ernst/Christina Costanza (Hg.): Personen im Web 2.0: Kommunikationswissenschaftliche, ethische und anthropologische Zugänge zu einer Theologie der Social Media. Göttingen, 48-72.

Marx, Konstanze (2012b): „Ich finde Dein Profil interessant“ - Warum virtuelle Erstkontakte auch für Linguisten interessant sind. In: Kristina Bedijs/Karoline H. Heyder (Hg.): Sprache und Personen im Web 2.0. Münster, 95-109.

Riemann, Viola (1999): Kontaktanzeigen im Wandel der Zeit. Eine Inhaltsanalyse. Opladen.

Schibli, Daniela (2014): Die Rubrik „Rendez-vous“ im Tages-Anzeiger - eine linguistische Analyse. Bachelor-Arbeit Universität Zürich.

Online unter: http://www.ds.uzh.ch/_files/uploads/studarb/39.pdf [19.9. 2016].

Skopek, Jan et al. (2009): Partnersuche im Internet. Bildungsspezifische Mechanismen bei der Wahl von Kontaktpartnern. In: Kölner Zeitschrift für Soziologie und Sozialpsychologie 61, 183-210.

Stolt, Birgit (1976): „Hier bin ich! - Wo bist Du?“ Heiratsanzeigen und ihr Echo analysiert aus sprachlicher und stilistischer Sicht. Kronberg i. Taunus.

Wyss, Eva L. (2011): Verlobungskorrespondenzen, Brautbriefe und Online-Flirts. Schriftliche Liebeskommunikation vom 19. Jahrhundert bis in die Internet-Ära. In: Martin Luginbühl/ Daniel Perrin (Hg.): Muster und Variation. Medienlinguistische Perspektiven auf Textproduktion und Text. Bern, 81-123. 
Bereitgestellt von | UZH Hauptbibliothek / Zentralbibliothek Zürich Angemeldet Heruntergeladen am | 06.04.18 10:52 